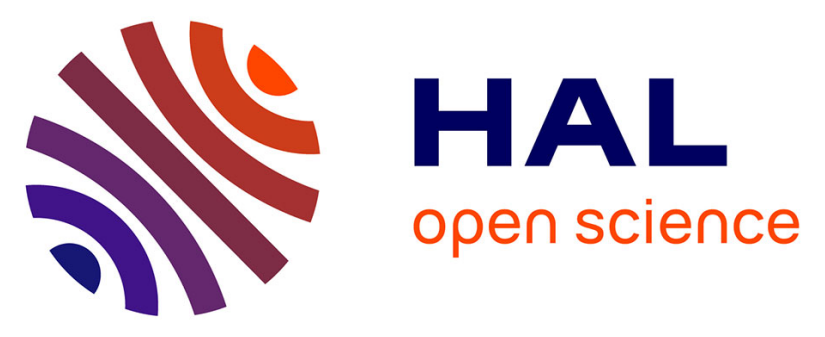

\title{
Determination of glass transition temperature using temperature dependent signal from a cryogenic photopyroelectric instrument
}

Allen Mathew, Fabrice Goutier, Benoit Escorne, Abdelaziz Elass, Gérard Louis, Abdelhak Hadj Sahraoui, Anne Baudot

\section{To cite this version:}

Allen Mathew, Fabrice Goutier, Benoit Escorne, Abdelaziz Elass, Gérard Louis, et al.. Determination of glass transition temperature using temperature dependent signal from a cryogenic photopyroelectric instrument. Thermochimica Acta, 2019, 676, pp.7 - 12. 10.1016/j.tca.2019.03.027 . hal-03485873

\section{HAL Id: hal-03485873 \\ https://hal.science/hal-03485873}

Submitted on 20 Dec 2021

HAL is a multi-disciplinary open access archive for the deposit and dissemination of scientific research documents, whether they are published or not. The documents may come from teaching and research institutions in France or abroad, or from public or private research centers.
L'archive ouverte pluridisciplinaire HAL, est destinée au dépôt et à la diffusion de documents scientifiques de niveau recherche, publiés ou non, émanant des établissements d'enseignement et de recherche français ou étrangers, des laboratoires publics ou privés.

\section{(ㄷ)(1) $\$$}

Distributed under a Creative Commons Attribution - NonCommerciall 4.0 International 


\title{
Glass Transition Study on Pure Glycerol Using Havriliak Negami Model from Temperature Dependent Photo Pyroelectric Signat Determination of Glass Transition Temperature Using Temperature Dependent Signal from a Cryogenic Photopyroelectric Instrument
}

\begin{abstract}
Allen Mathew ${ }^{a}$ (Corresponding author), Fabrice Goutier ${ }^{a}$, Benoit Escorne ${ }^{b}$, Abdelaziz Elass $^{a}$, Gérard Louis $^{c}$, Abdelhak Hadj Sahraoui $^{a}$, Anne Baudot $^{c}$

${ }^{a}$ UDSMM, Université du Littoral Côte d'Opale (ULCO), 145 avenue Maurice Schumann, Dunkerque 59140, France. (allen_mathew88@yahoo.com, fabrice.goutier@univ-littoral.fr, elass@univ-littoral.fr, hadj@univ-littoral.fr)

${ }^{b}$ Pôle de Recherche "Environnement, Milieux Littoraux et Marins" (EMLM), ULCO, 145 avenue Maurice Schumann, Dunkerque 59140, France. (escorne@univ-littoral.fr)
\end{abstract}

${ }^{c}$ Université Paris Descartes, Sorbonne Paris Cité - INSERM U1148, UFR biomédicale, 45 rue des Saints-Pères, Paris 75270, cedex 6, France. (gerard.louis@parisdescartes.fr, anne.baudot@parisdescartes.fr)

March 16, 2019

\footnotetext{
Highlights

A photopyroelectric device for $90 \mathrm{~K}$ to ambient thermal studies was constructed.

Temperature controlled LED was used to heat the sensor/sample interface.

The thermal diffusion equations for FPPE configuration were solved.

Sensor used was $\mathrm{LiTaO}_{3}$ with an ITO layer on one side and gold on other.

The glass transition temperature was obtained directly from the effusivity.
} 


\begin{abstract}
Herein, we report the construction of a low temperature photo pyroelectric (PPE) instrument using which the glass transition temperature $\left(T_{g}\right)$ of a well known glass former (glycerol) is determined via temperature dependent PPE signal. Havriliak Negami (HN) model was used to analyze the PPE signal. As expected, relaxation time for glycerol follows Vogel Fulcher Tammann (VFT) model. The $T_{g}$ was determined from the HN and VFT parameters. Obtained results were compared with previously published data.
\end{abstract}

Keywords: Photo Pyroelectricity, Cryogenic instrumentation, Havriliak Negami model, Glass transition temperature, Glycerol 


\section{INTRODUCTION}

Understanding the glass transition phenomenon is one of the most challenging problems in the field of materials science in the 21 st century. Various techiniques have been used to study the glass transition phenomenon, for instance, dielectric measurements [1], nuclear magnetic resonance (NMR) technique [2], Differential Scanning Calorimetry (DSC) and modulated DSC [3, 4], Adiabatic Scanning Calorimeter (ASC) [5], photothermal studies [6-8], viscosity studies $[9,10]$, etc. A glassy state is associated with a structural relaxation time $(\tau)$ of $100 \mathrm{~s}$ [11] or a viscesity $(\eta)$ of $10^{12} \mathrm{~Pa}$ s or $10^{13} \mathrm{P}$. The time scale of $\tau$ increases drastically on rapid cooling, same happens for the viscosity. The dependence of $\tau$ on temperature can be defined using different theoretical models like Vogel Fulcher Tammann (VFT)[12, 13], Bässler-Avramov (BA)[14, 15], Waterton-Mauro (WM or MYEGA)[16, 17], Critical-like (Crit)[18, 19] etc.

Photothermal techniques have been used for decades for the non destructive analysis of materials. Photo pyroelectric (PPE) technique is a very powerful and simple photothermal technique to study the thermal properties of materials. One of the main advantage of PPE over other methods is that it can study all the thermal properties. As the relaxation time is temperature dependent, it is interesting to study the thermal properties such as thermal effusivity, thermal diffusivity, thermal conductivity and specific heat capacity. Hot wire, the laser flash and the photothermal methods are used for the determination of thermal properties in liquids [20]. Among the photothermal techniques, the photopyroelectric (PPE) methodology is the most appropriate for a complete thermal characterization of liquid and pasty samples [21]. The major advantages of the photopyroelectric method are its simplicity, high sensitivity, non-destructive character and its easy adaptation to the experimental restrictions imposed by the theoretical requirements [22]. The high signal to noise ratio of PPE for a wide range of frequency (10 $\mathrm{mHz}-100 \mathrm{kHz}$ ) [8] enables the detection of smaller temperature variation, thereby increasing its application in the study of phase transition. R. Ivanov et al. studied thermal effusivity using electropyroelectric technique.

For phase transition study using PPE technique, we study thermal effusivity Relaxation study can be performed using PPE technique from thermal effusivity [23] which is expressed in unit, $W s^{1 / 2} m^{-2} K^{-1}$ and is given by:

$$
e=\sqrt{\rho c k}
$$

where $\rho$ is the density, $\mathrm{c}$ is the heat capacity and $\mathrm{k}$ is the thermal conductivity. From thermal effusivity measurement, information about the frequency dependence of heat capacity (c) can be retrieved. In the particular case of liquids, the photopyroelectric technique in the front detection configuration has been emerged as one of the most useful methods for measurement of effusivity [24]. Surprisingly, no study was reported concerning the determination of glass transition temperature directly from the temperature dependent effusivity using PPE. 
In PPE technique, a frequency dependent temperature response is obtained from the pyroelectric material when a modulated heat is supplied. The frequency dependence behave in a similar way as classical relaxing susceptibilities like elastic modulus and dielectric permitivity. Generally, this frequency dependent response at the vicinity of relaxation is studied using the Havriliak Negami (HN) model. Contrarly, here we analyse the temperature dependent signal directly using HN model to investigate the glass transition in glycerol. For that, the relaxation peaks observed in the temperature evolution of thermal effusivity is analysed using HN model.

The objective of the study is to extend the PPE Calorimetry to the low temperature range (90K to ambient) in order to determine the glass transition temperature from the temperature relaxation of effusivity. To do that, we built a cryogenic PPE instrument. To compare with literature, we selected a well-known glass-former, glycerol. It is used as cryoprotectants (CPAs) in different cryopreservation processes. CPAs are the necessary essential ingredients to protect the cells from freezing damage.The colligative property of glycerol [25] helps to bind with water, leading to an increased viscosity of the body fluids and thus reducing the availability of nucleating sites. By varying the concentration and cooling rate of the glycerol-cell mixture, it is possible to obtain a wholly amorphous state [26] which is essential for cell survival. Studying a well known sample like glycerol helped to calibrate the newly constructed PPE instrument.

\section{MATHEMATICAL MODELING OF A PPE SIGNAL}

\subsection{PPE signal}

The pyroelectric materials have the ability to generate a temporary voltage due to the change in the electric polarization because of the changing heat flux. This property is called pyroelectric effect. In a PPE setup, the changing heat flux is realised obtained using a modulated light. For the measurements of the thermal effusivity (e) of glycerol, we used the front detection photo pyroelectric technique (FPPE) $[24,27,28]$ with a semi transparent pyroelectric sensor $[29,30]$ as shown in figure 1 .

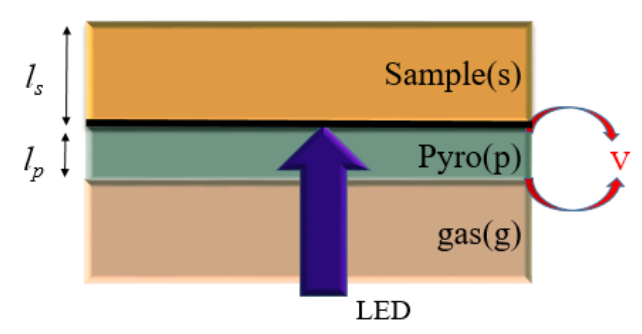

Figure 1: FPPE configuration with semi transparent sensor. Heat is produced at the sample/pyro interface and flows to both pyro sensor and sample. $l_{s}$ and $l_{p}$ are the thickness of sample and pyroelectric sensor respectively.

The normalized expression for voltage generated across pyro sensor for this configuration 
is given by [23]:

$$
V(f, T)=\frac{1-e^{-2 \sigma_{p} l_{p}}}{\left(1+b_{s p}\right)\left[1+R_{s p} e^{-2 \sigma_{p} l_{p}}\right]}
$$

where $\sigma$ is the complex wave number given by $\sigma=(1+i) \frac{1}{\mu}$, where $\mu$ is the thermal diffusion length (normally expressed in $\mu m$ ) and is given by: $\mu=\sqrt{\frac{\alpha}{\pi f}}$ [31], where $\alpha$ is the thermal diffusivity and $\mathrm{f}$ is the modulation frequency. $R_{i j}$ represents the reflection coefficient of the thermal waves between two media i and $\mathrm{j}$ (where $\mathrm{i}, \mathrm{j}$ can be any layer: sample(s) or pyro(p) or gas(g)): $R_{i j}=\frac{b_{i j}-1}{b_{i j}+1}$ where $b_{i j}=\frac{e_{i}}{e_{j}}$ [32] which gives $b_{s p}=\frac{e_{s}}{e_{p}}$ [32] and $R_{s p}=\frac{b_{s p}-1}{b_{s p}+1}$ [32], where $e_{s}, e_{p}$ are the effusivities of sample and pyro sensor respectively. $t_{p}$ is the thickness ef pyro senser.

For a thermally thick pyro sensor $\left(\mu_{p}<l_{p}\right)$ and sample $\left(\mu_{s}<l_{s}\right)$, the normalized expression for $\mathrm{V}$ is given by [23]:

$$
V(f, T)=\frac{1}{1+b_{s p}}=\frac{1}{1+\frac{e_{s}}{e_{p}}}
$$

To verify that $\mathrm{LiTaO}_{3}$ is thermally thick, we calculated the thermal diffusion length taking into account the temperature evolution of diffusivity of $\mathrm{LiTaO}_{3}$ from the literature [33] and we found that sensor was thermally thick in our working frequency and temperature range.

Thermal effusivity is given by the equation 1. The quantity in which relaxation occurs is the dynamic heat capacity (c). It has been reported in literature that frequency dependence of the thermal conductivity $(\mathrm{k})$ is negligible in comparison with that of heat capacity $[8,34]$. The frequency dependence of heat capacity of glass formers is complex. This can be described by the Havriliak-Negami (HN) empirical model [35] and is given by the expression:

$$
c(\omega, T)=c_{\infty}+\left[\frac{c_{0}-c_{\infty}}{\left(1+(i \omega \tau)^{\alpha}\right)^{\beta}}\right]
$$

Where $\omega$ is the angular frequency and is given by $\omega=2 \pi \nu$, where $\nu$ is the frequency. $c_{0}$ and $c_{\infty}$ are constants. $\alpha$ and $\beta$ are always in the range $0 \geq \alpha \geq 1$ and $0 \geq \beta \geq 1$ respectively.

Simulation of temperature evolution of amplitude and phase of the PPE signal using the equation 3 for pure glycerol at different modulation frequencies has been performed. Here VFT model is used to define the temperature dependence of $\tau$. The simulated normalized PPE signal during a temperature scan is shown in figure 2.

From the amplitude and phase of the PPE signal we can calculate the effusivity $\left(e_{s}\right)$ of the sample by using the equation 3 , provided we must know the effusivity of the pyroelectric sensor $\left(e_{p}\right)\left(\mathrm{LiTaO}_{3}\right.$ in our case). The dependence of effusivity on temperature for $\mathrm{LiTaO}_{3}$ was reported by Oleaga et al [33]. From equation 1, it is evident that the dynamic heat capacity can be studied directly from the squared effusivity. Therefore, the equation 4 can be rewritten as 

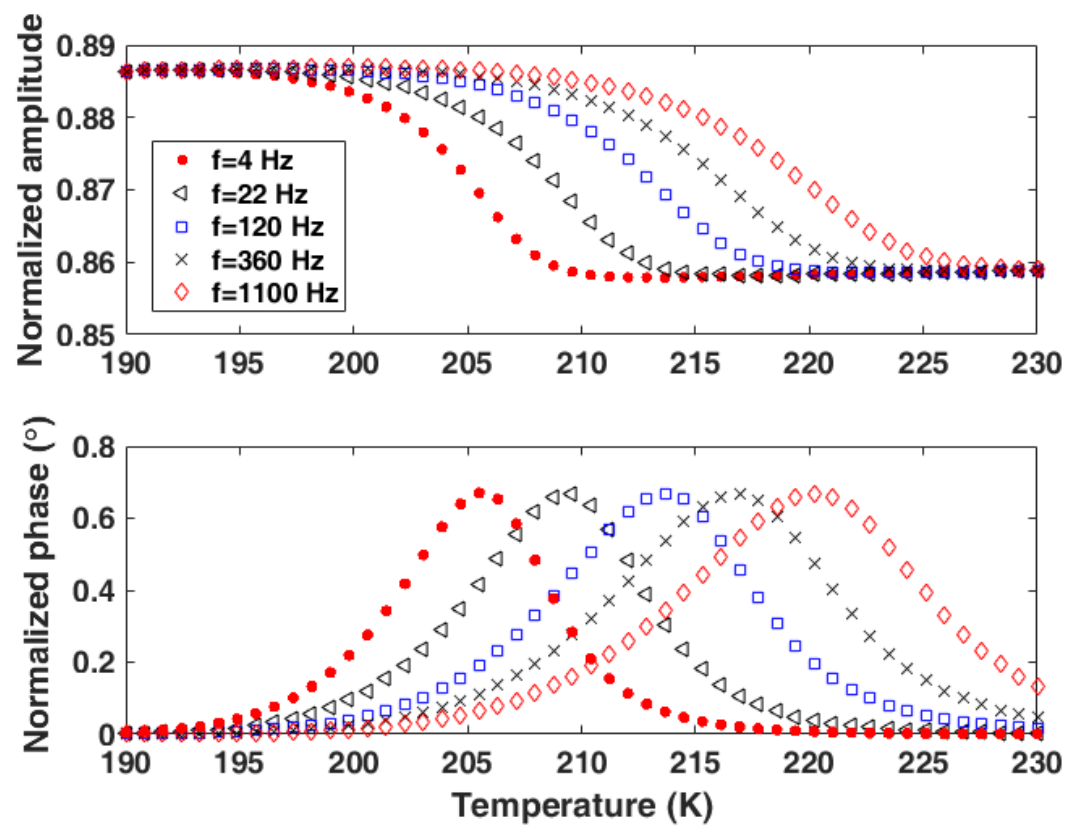

Figure 2: Simulation of temperature evolution of amplitude and phase of the PPE signal using the equation 3 for pure glycerol at different modulating frequencies. Here VFT model is used to define the temperature dependence of $\tau$.

follows

$$
e_{s}^{2}(\omega, T)=e_{s \infty}^{2}+\left[\frac{e_{s 0}^{2}-e_{s \infty}^{2}}{\left(1+(i \omega \tau)^{\alpha}\right)^{\beta}}\right]
$$

Figure 3 shows a simulation of the temperature evolution of real and imaginary parts of squared effusivity for different modulating frequencies derived from the PPE signal shown in the figure 2 .

Generally, the HN equation is used in frequency domain to study the relaxation mechanism, as in the case of dielectric spectroscopy [36] which has been used to study glass transition phenomenon. In our case, HN equation (5) has been adopted in temperature domain to study the relaxation mechanism. Here $\tau$ is the only temperature dependent parameter. $\omega$ is fixed because a temperature scan is performed at fixed frequency and $\tau$ varies with temperature. For glycerol, the behavior of $\tau$ as function of temperature is given by the VFT model[37, 38]:

$$
\tau(T)=\tau_{\infty} \exp \left(\frac{D T_{0}}{T-T_{0}}\right) \quad \text { where } D=B / T_{0}
$$

By incorporating equation (6) into $\mathrm{HN}$ equation, it is possible to solve all the seven parameters, $\alpha, \beta, e_{s 0}^{2}, e_{s_{\infty}}^{2}, \mathrm{~B}, T_{0}$ and $\tau_{\infty}$, at a time using a "7-parameter fit", which will be explained in the coming section. Once the VFT parameters are known, $T_{g}$ can be determined by using the glass transition convention, $\mathrm{T}=T_{g}$ when $\tau=100 \mathrm{~s}[11]$. 

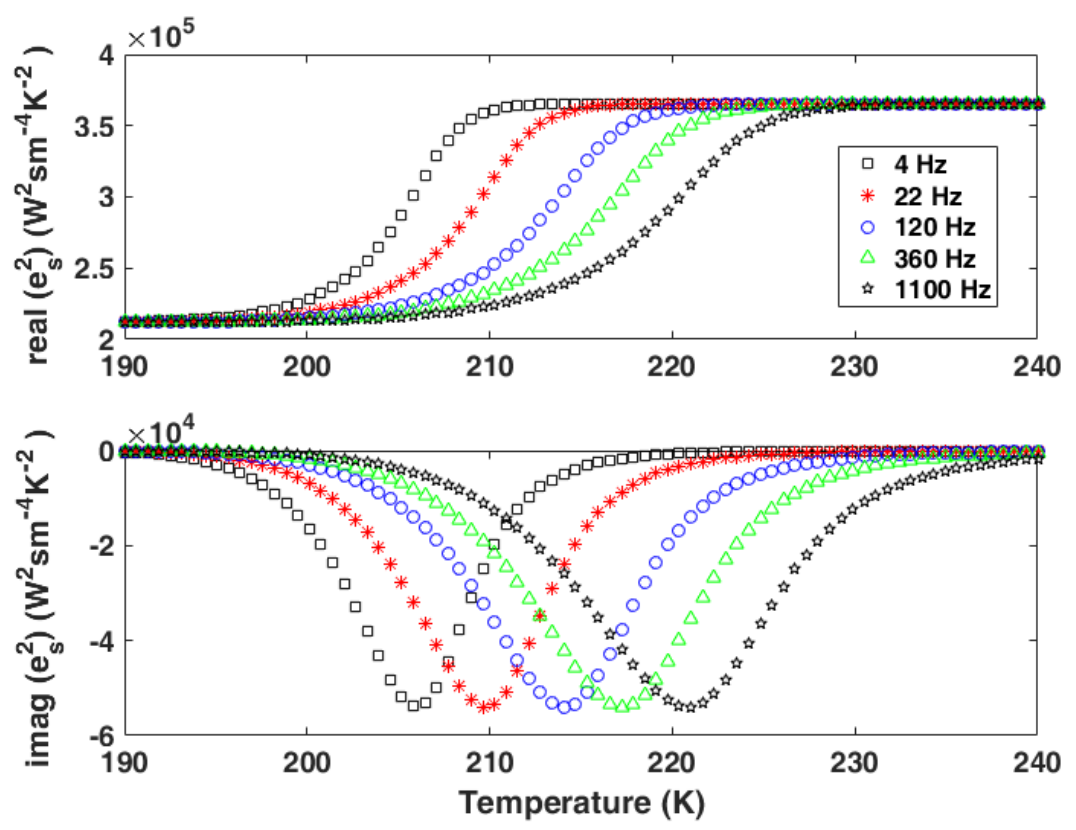

Figure 3: Simulation of temperature evolution of real and imaginary parts of squared effusivity for pure glycerol at different modulating frequencies.

\section{EXPERIMENT}

To measure the thermal effusivity of glycerol we built a low temperature PPE instrument. The whole setup can be described in three parts: PPE cell, Illumination and temperature controller and Data acquisition.

\subsection{PPE cell}

\subsubsection{Cell Design}

A cell was designed to study precisely the thermal effusivity of liquid samples as function of temperature. Liquid nitrogen was used for cooling the cell till $-180{ }^{\circ} \mathrm{C}$. The pyroelectric materials that we used were piezoelectric also The pyroelectric materials that we used were also piezoelectric [39]. Therefore, in addition to the pyroelectric signal, they generate a piezoelectric signal as a response to mechanical stress. The piezoelectric effect dominates due to the mechanical stress generated by the flow of liquid nitrogen when it comes in direct contact with pyroelectric sensor. Therefore, it was very necessary to isolate the sensor from the nitrogen flow. Basically the cell was made in aluminum with two chambers, A and B, shown in figure 4.

Chamber A hold the pyroelectric sensor and the sample while chamber B was used for the circulation of liquid nitrogen. Inlet and outlet valves for liquid nitrogen were connected to chamber B. The chamber A was fixed inside the chamber B. The chamber A offered complete isolation of the sensor and sample from liquid nitrogen flow inorder in order to avoid the afore- 


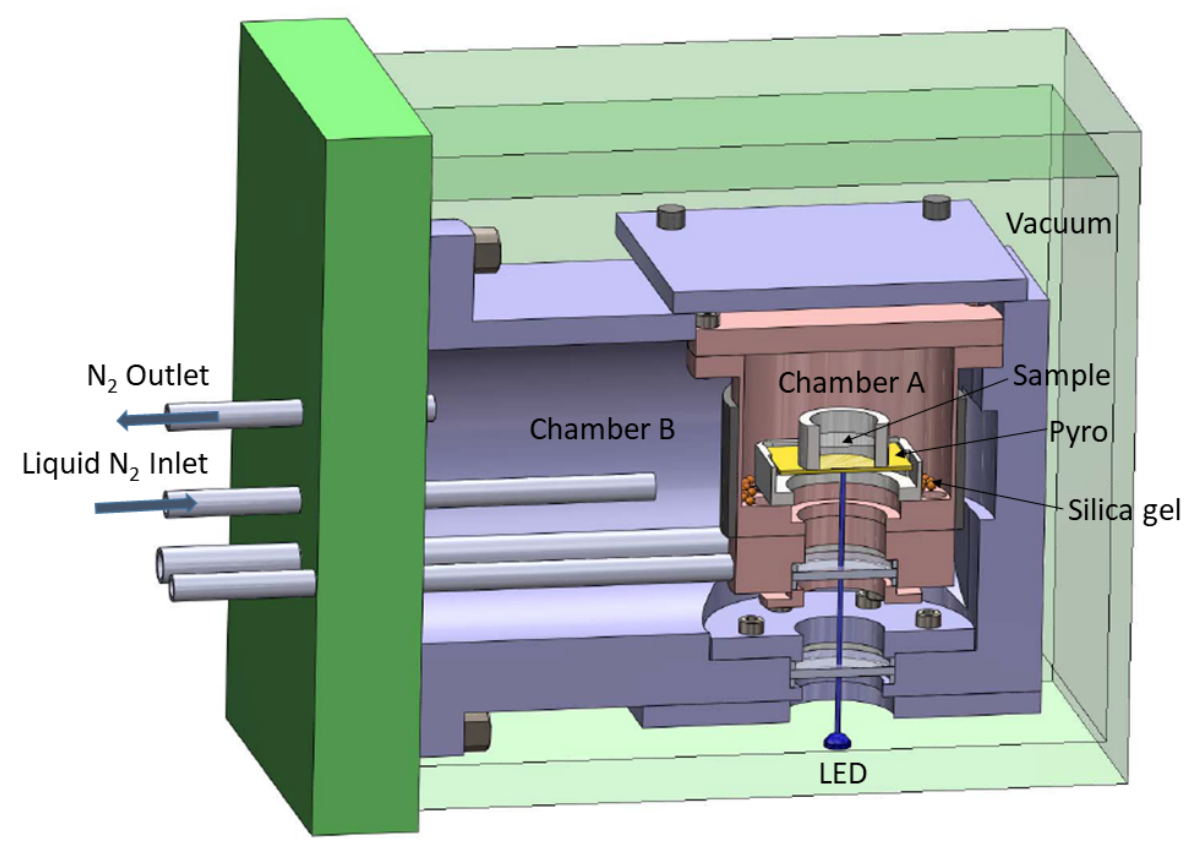

Figure 4: Schematic representation of the PPE cell.

mentioned afore mentioned piezoelectric dominance.

The chamber A was adapted for both PPE configurations. We used a structure made from polytetrafluoroethylene (PTFE), commonly known as Teflon ${ }^{T M}$, to hold the sample in contact with the pyroelectric sensor. The sample was placed above the sensor as shown in figure 4 so that the sample could maintain a better contact with the sensor throughout the low temperature experiments. The structure allowed to add samples without displacing the sensor. This was because each measurement was a set of two experiments, first without sample and the second with sample. A normalization was done with signal measured with sample to the one without sample thereby nullifying the dependence of the electronic and illumination contributions. Maintaining atmospheric pressure inside the chamber A was very important and this was done by introducing an opening to the surroundings without affecting the nitrogen flow.

The illumination was given from bottom of the cell, because when the sample became opaque during freezing, the radiation couldn't reach the pyro sensor. Glass windows were used for the light entrance. The glass was fixed with PTFE O-rings between aluminum. The whole cell was isolated from the surroundings by keeping it in vacuum. Cold leaks were detected by checking the vacuum level. Cryogenic vacuum grease was used at all the contacts to avoid cold leaks.

\subsubsection{Pyroelectric Sensors Used for Measurement}

A square shaped $\mathrm{LiTaO}_{3}$ sensor (OPTON LASER INTERNATIONAL) with a thickness of $300 \mu \mathrm{m}$ and 30x30 $\mathrm{mm}^{2}$ surface area with gold coated on both sides was used for the effusivity measurements at the beginning. Here air-sensor interface was illuminated. The thickness of the 
sensor limited our working frequency range because the heat wave has to traverse the whole thickness of the pyro sensor to reach the sample which is on the other side. To overcome this limitation, we used the semi transparent pyro sensor where the sample-sensor interface was illuminated. A pyro sensor with both sides coated with opaque electrode limits the modulation frequency because the heat wave has to penetrate the whole thickness of pyro to reach the sample. Therefore, the modulation frequency must be carefully chosen to satisfy this condition. Semi transparent pyro sensor helps to extend the range of modulation frequency. While using a semi transparent pyro, the light is incident at the pyro-sensor interface and the problem of limiting the modulation frequency is solved. A semi-transparent $\mathrm{LiTaO}_{3}$ with one side coated with indium tin oxide (ITO) and other side with gold was used for low temperature measurements. The ITO was deposited by SOLEMS (ITO sol 30) on a $\mathrm{LiTaO}_{3}$ from OPTON LASER INTERNATIONAL. The $\mathrm{LiTaO}_{3}$ dimensions were $500 \mu \mathrm{m}$ in thickness and 30x30 mm² surface area. Copper wires used for connections were fixed using silver paint (Agar scientific).

\subsubsection{Temperature Measurements}

Temperature measurements were done using Pt1000 sensors in four wire connection using two multimeters: Keithley 3706 and Keithley 2700. Four wire connection offers better reliability and accuracy. For low temperature measurements, Pt1000 (Class Y) sensors were used. Multimeter K2700 was dedicated for the temperature measurements inside chamber A. All other temperatures were measured using K3706. The reason for a dedicated multimeter for measurements inside the chamber A was due to the generation of noise in the LOCK-IN amplifier signal because of the near proximity of pyro sensor with the Pt1000 sensor. The signal from the LOCK-IN amplifier was disturbed when all Pt1000 sensors were triggered using the multimeter K3706. So the temperature inside the chamber A was measured using the K2700 multimeter after the acquisition of the PPE signal by the LOCK-IN.

It was always a problem to measure the sample temperature accurately because the sensor could not be in contact with the sample as this induced noise. The sensor dedicated to the measurement of the sample temperature was freely suspended in air (T1) in close proximity to the sample-sensor combination. To reduce the error, for each unknown sample, first we made an experiment with another sensor (T2) put inside the cup filled with the same quantity of sample which would be used for the effusivity measurements. There was a difference between these two temperatures while heating and is given by $\Delta T=T 1-T 2$. Therefore, the temperature of the sample was corrected using $\Delta T$ and is given by $T 2=T 1-\Delta T$.

Temperature of the chamber A was maintained by using a heating coil wrapped around it and controlled by a PID. Power delivered to the PID was always the same AC line voltage (220-240V). This PID setup consisted of a step down transformer (220 230 (AC) V to 3224 (DC) V) to power a MODBUS communication interface and a solid state relay. Sensors were fixed on the outer surface of the chamber A. During this process, LABVIEW program was 
controlling the solid state relay via MODBUS communication and delivering the line voltage to the heating ring around the cell. The heating ring has a resistance of $220 \mathrm{ohms}$ and a power rating of $220 \mathrm{~W}$. The temperature of the cell (T) was controlled with a precision of $\pm 0.01{ }^{\circ} \mathrm{C}$.

\subsection{Illumination and Temperature Controller}

A periodic heating is necessary for the pyroelectric sensor to generate a signal. It was done using an LED, very cost efficient compared to a laser. To power the LED, a driving circuit was used which took input from a power supply and the LOCK-IN amplifier provided the reference frequency for a periodic illumination. We have observed that the heat generated in the transistor in the driving circuit resulted in a change in the phase of the signal from the pyro sensor. To avoid this, a PID was used to regulate the temperature of the transistor. A peltier was used and its one side was fixed to the transistor, which should be controlled at a fixed temperature and the other side to a heat sink. The peltier was powered by a TTi CPX200D power supply. LXi interfacing was used between the TTi power supply and computer. Controlling and interfacing were done with LABVIEW program. The best possible Ziegler Nichols parameters were found to set the PID with a precision of $\pm 0.01{ }^{\circ} \mathrm{C}$.

The main disadvantage of LED was the influence of its junction temperature on the intensity of the light. The amplitude of the signal kept decreasing as the junction temperature increased. The solution to this problem was to control the junction temperature perfectly. Many attempts were tried and finally a perfect heat sink was attached to the cathode terminal of the LED, and the temperature was controlled using a PID just like the same mentioned earlier. This was also set to a precision of $\pm 0.01^{\circ} \mathrm{C}$.

\subsection{Data Acquisition}

The pyroelectric signal was measured by the LOCK-IN amplifier from Signal Recovery (model 7124). The measurements were performed in current mode with manual settings for sensitivity, AC gain and time constant. The data was sent to a computer and all interfacing were done using LABVIEW programming. The length of the BNC cables and connectors were optimized to avoid noise and protected from electromagnetic interference using faraday protection.

\subsection{Low Temperature Experiments}

For low temperature experiments, we chose the FPPE configuration with a semitransparent $\mathrm{LiTaO}_{3}$, coated with ITO on one face, and gold on other was in contact with the sample. The light entered through the transparent ITO and heated the sensor-sample interface. The system was cooled from ambient temperature to $-196{ }^{\circ} \mathrm{C}$ at a rate of $6{ }^{\circ} \mathrm{C} \mathrm{min}^{-1}$ by circulating liquid 
nitrogen and hold at this temperature for 10 min to avoid any temperature gradient in the sample. The flow was stopped and the cell was allowed to heat to ambient temperature at a rate around $1{ }^{\circ} \mathrm{C} \min ^{-1}$. Data acquisition was done from $-180{ }^{\circ} \mathrm{C}$ to $20{ }^{\circ} \mathrm{C}$ at every second while heating. Fast cooling of $\mathrm{LiTaO}_{3}$ results in building up of surface charges on it and one should be careful when establishing a connection to the LOCK-IN amplifier. A shorting BNC has been used to discharge the connection leads before connecting to the LOCK-IN amplifier.

Temperature scans were performed at fixed frequencies. For normalization, it was important to perform an experiment without sample and this must be performed prior to the one with sample so that sample could be added without displacing the sensor. It was possible to observe the temperature range where the heat capacity undergoes a relaxation. This relaxation has been seen as a drop in the amplitude and a maximum in the phase of the signal. As observed by other researchers [34] we have also observed that the temperature range, where the relaxation happened, was shifting to higher values when the modulation frequency was increased. Our frequency range was from $22 \mathrm{~Hz}$ to $1 \mathrm{kHz}$. The reason for the low frequency limit was to follow the thermally thick condition. High frequency was limited to $1.1 \mathrm{kHz}$ to avoid noise. This noise can be from the quality of the ITO layer.

\subsection{Sample Used}

Before using, glycerol from ALPHA AESAR, Spectrophotometric grade, 99+\%, was kept for 15 hours in an oven at $40^{\circ} \mathrm{C}$. The percentage of relative humidity in this oven was always below $15 \%$ which lead to a water content in glycerol less than $5 \mathrm{wt} \%$ [40]. Silica gel was put all around the sample (see figure 4) to decrease the atmospheric water content in the chamber A. At the beginning of the experiment the cell was flushed with gaseous nitrogen from the liquid nitrogen tank. During cooling, the temperature of the sample was always above the temperature of the cell air and the temperature of the aluminum was the lowest. The surfaces acted like a cold finger where residual water condensed first and then solidified below $0^{\circ} \mathrm{C}$. When the temperature of the sample reached $0^{\circ} \mathrm{C}$, the cell air temperature was around $-40^{\circ} \mathrm{C}$ and the temperature of the cell surface was $-60^{\circ} \mathrm{C}$. It means that during the experiment, the percentage of relative humidity in the surroundings of the glycerol was very low and clearly below $10 \%$. Therefore, it was assumed that the water content in the sample during the measurements was below $5 \mathrm{wt} \%$.

\section{RESULTS}

\subsection{Squared Effusivity from PPE}

The normalized amplitude and phase of pure glycerol at $22 \mathrm{~Hz}$ is shown in figure 5 .

The relaxation was observed on both amplitude and phase of the signal as explained earlier. 


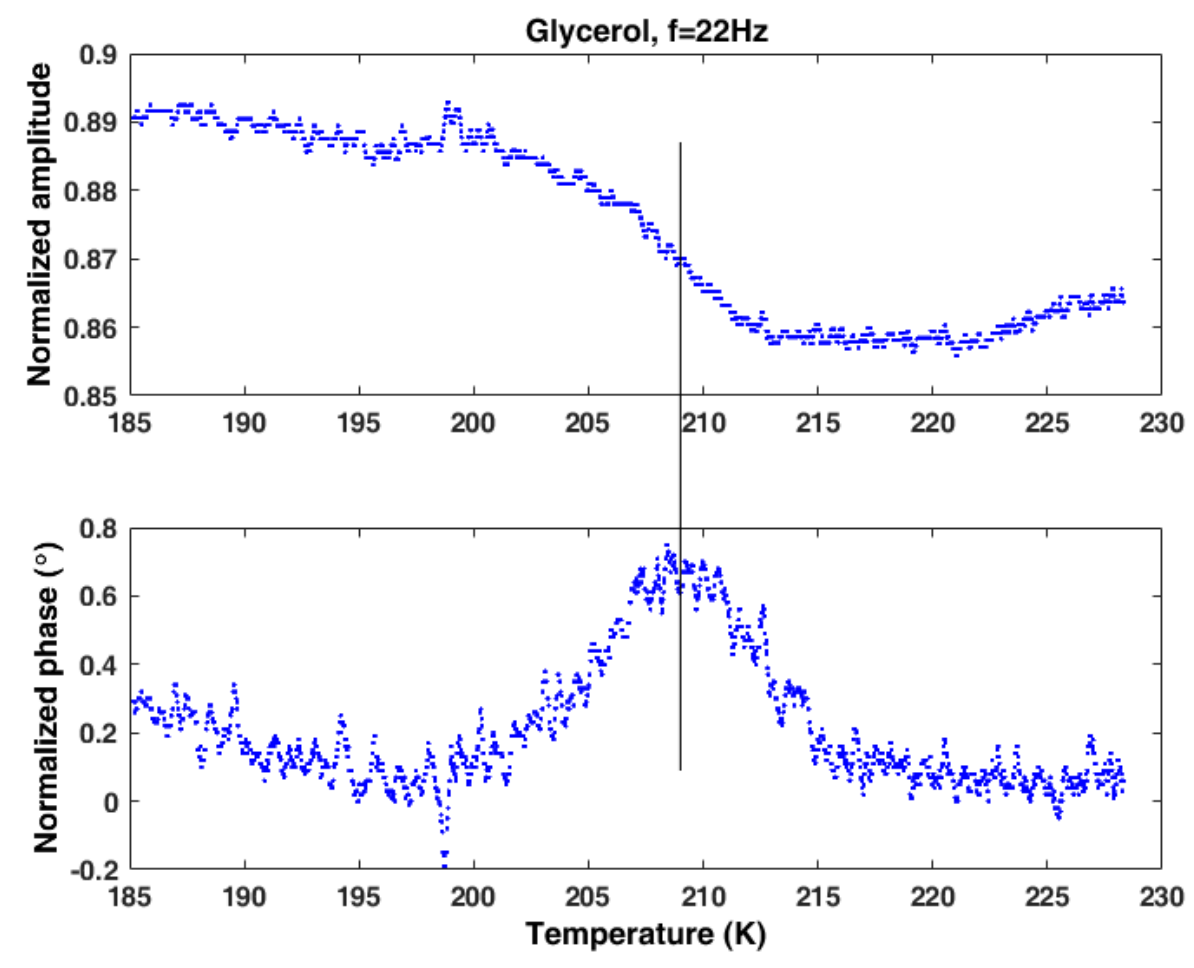

Figure 5: Normalized amplitude and phase of pure glycerol during a temperature scan at a fixed frequency of $22 \mathrm{~Hz}$ using a $500 \mu \mathrm{m} \mathrm{LiTaO}_{3}$ as sensor.

The amplitude suffered a drop and phase attained a peak and relaxed during the $\alpha$ relaxation. The temperature at the point of inflection in the amplitude and maximum of the phase corresponded. However, Figure 2 shows that the normalized amplitude cannot increase with increase in temperature and that the normalized phase should tend to zero far from the relaxation. In the Figure 5 we can see a slight deviation from this trend. Such deviation can appear in the signal but is not a problem if it is outside the relaxation region. So the results were used in the narrow temperature range $202-216 \mathrm{~K}$ which was the alpha relaxation region.

Using equation 3, the squared effusivity of pure glycerol as a function of temperature was calculated. The temperature evolution of its real and imaginary parts is shown in figure 6 . The relaxation of heat capacity can be observed in this graph in both real and imaginary parts. A discrepancy is visible between the simulation (figure 3) and the squared effusivity plot (figure 6). This is due to the above mentioned deviation in the experimental data (figure 5).

\subsection{Fit Procedure}

\subsection{Determination of $\mathbf{T g}$}

The temperature dependence of $\tau$ for glycerol is given by VFT model. Therefore, $\tau$ in the $\mathrm{HN}$ equation was replaced by VFT equation and then we fitted the temperature evolution of real and imaginary parts of $e_{s}^{2}$ using equation 5 for determining all 7 parameters $(4 \mathrm{HN}: \alpha, \beta$, $e_{s 0}^{2}, e_{s \infty}^{2}$ and 3 VFT: B, $T_{0}$ and $\left.\tau_{\infty}\right)$. 


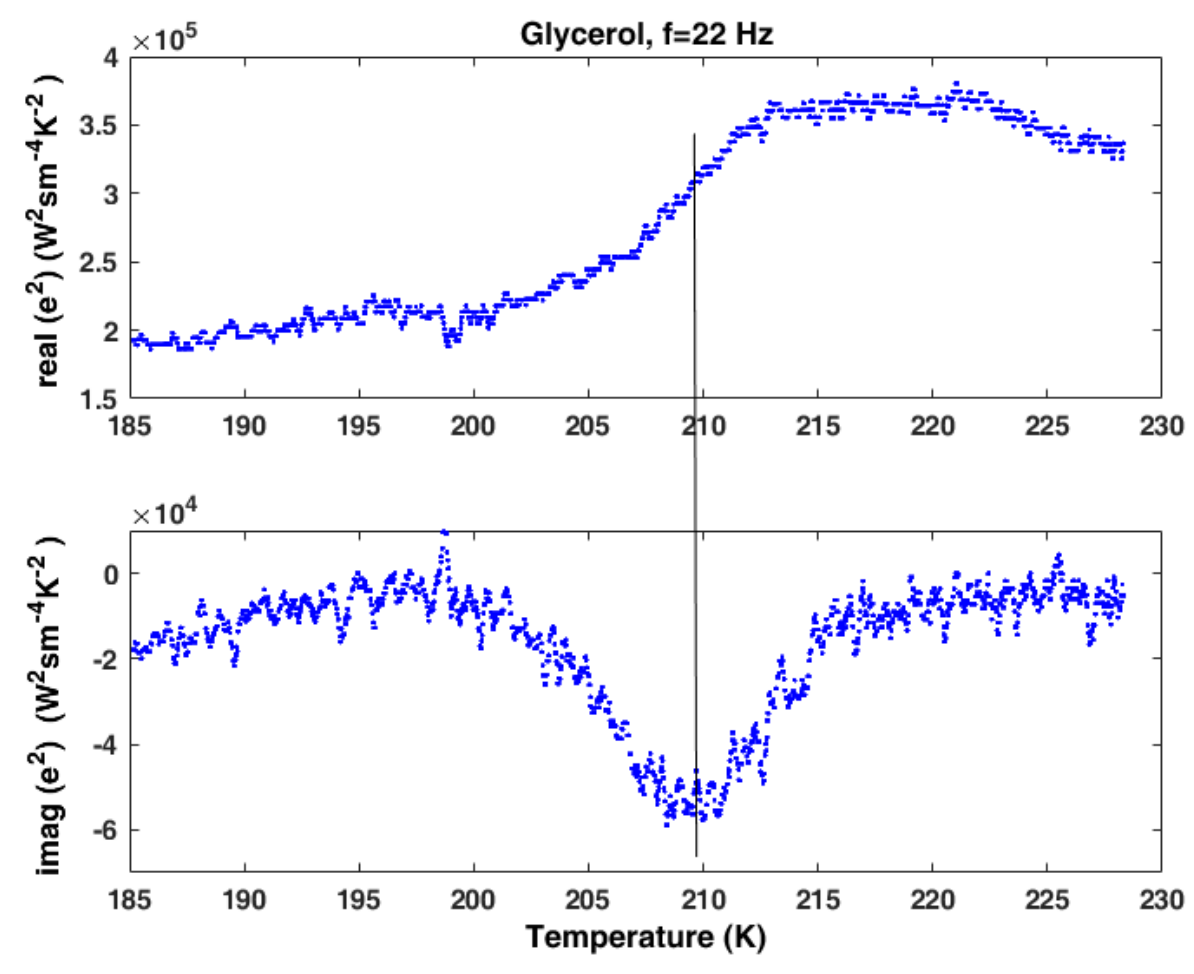

Figure 6: Real and imaginary parts of squared effusivity versus temperature for pure glycerol at a fixed frequency of $22 \mathrm{~Hz}$.

For the fit, only the $\alpha$ relaxation region was considered and this was a narrow temperature range. Figure 7 shows the best fitted real and imaginary parts of $e_{s}^{2}$. The circular legend represents the experimental data and the smooth one represents the best fitted curve.

The same procedure was applied for the experimental data from other frequencies. In a similar fashion, temperature scans at different fixed frequencies, say, $120 \mathrm{~Hz}$ and $1100 \mathrm{~Hz}$ were performed.

The normalized amplitude and phase of temperature scans at different frequencies $(22 \mathrm{~Hz}$, $120 \mathrm{~Hz}, 1100 \mathrm{~Hz}$ ) performed are shown in figure 8. Considering the easiness of representation, all normalized amplitude curves are moved to 1 and all normalized phase curves are moved to 0 at $235 \mathrm{~K}$. The relaxation can be observed in the amplitude and phase from each frequency scan. It is evident that the peak in the phase moves to higher temperature as the scanning frequency is increased, as observed by other researchers [34] and in the simulation shown in figure 2.

The fit has been performed on each frequency raw data and the best fitted parameters are given in table 1 .

Once the VFT parameters were known, using the glass transition convention [11] $(\tau=100 \mathrm{~s}$ at $T_{g}$ ), Tg of pure glycerol was determined from each frequency and the mean value is reported in table 2. Obtained result is found to be in good agreement with literatures. The error range +/-6 K, can be due to the experimental uncertainty and the water content in glycerol. 

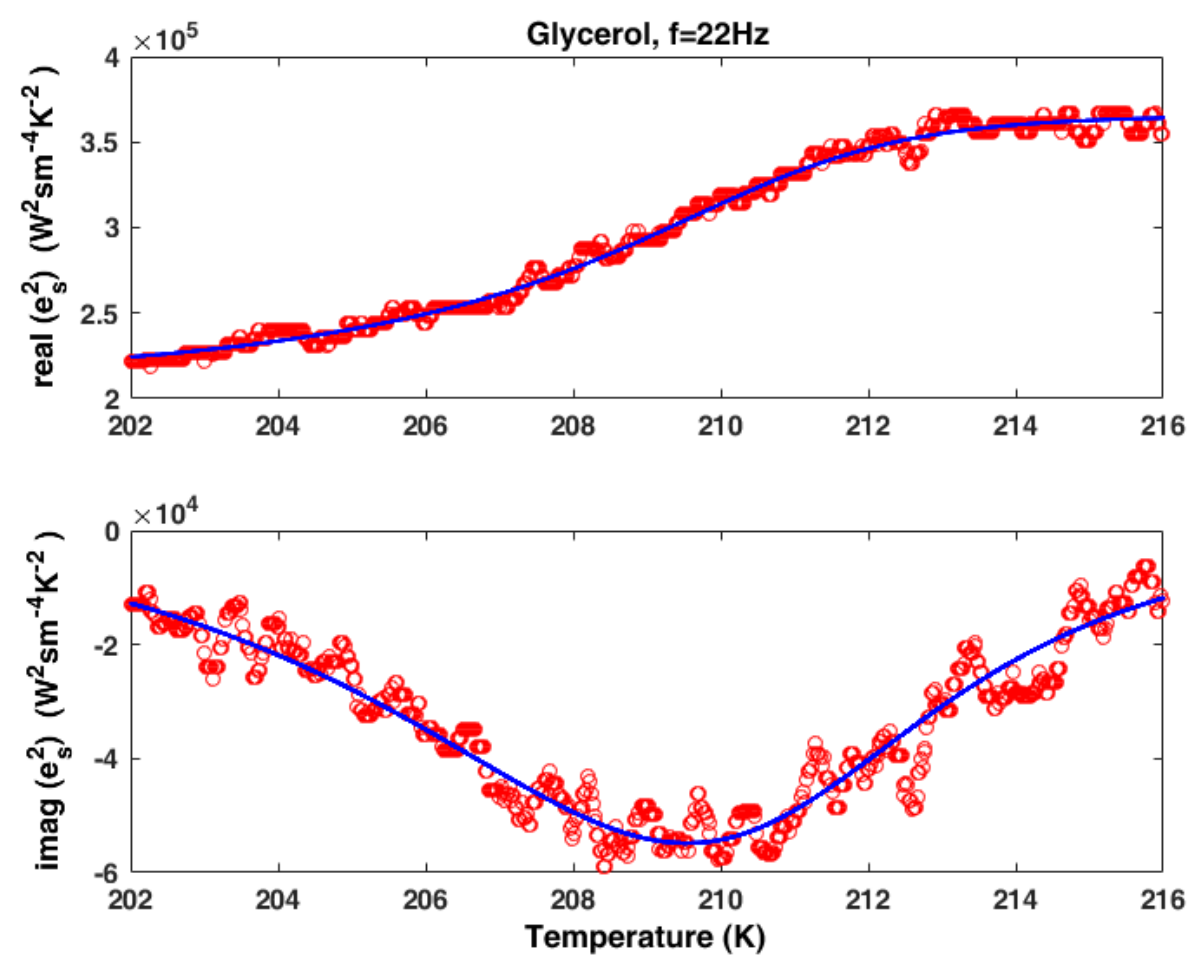

Figure 7: Fitting done on the real and imaginary parts of squared effusivity versus temperature for pure glycerol using Havriliak-Negami model and 7-parameter fit.
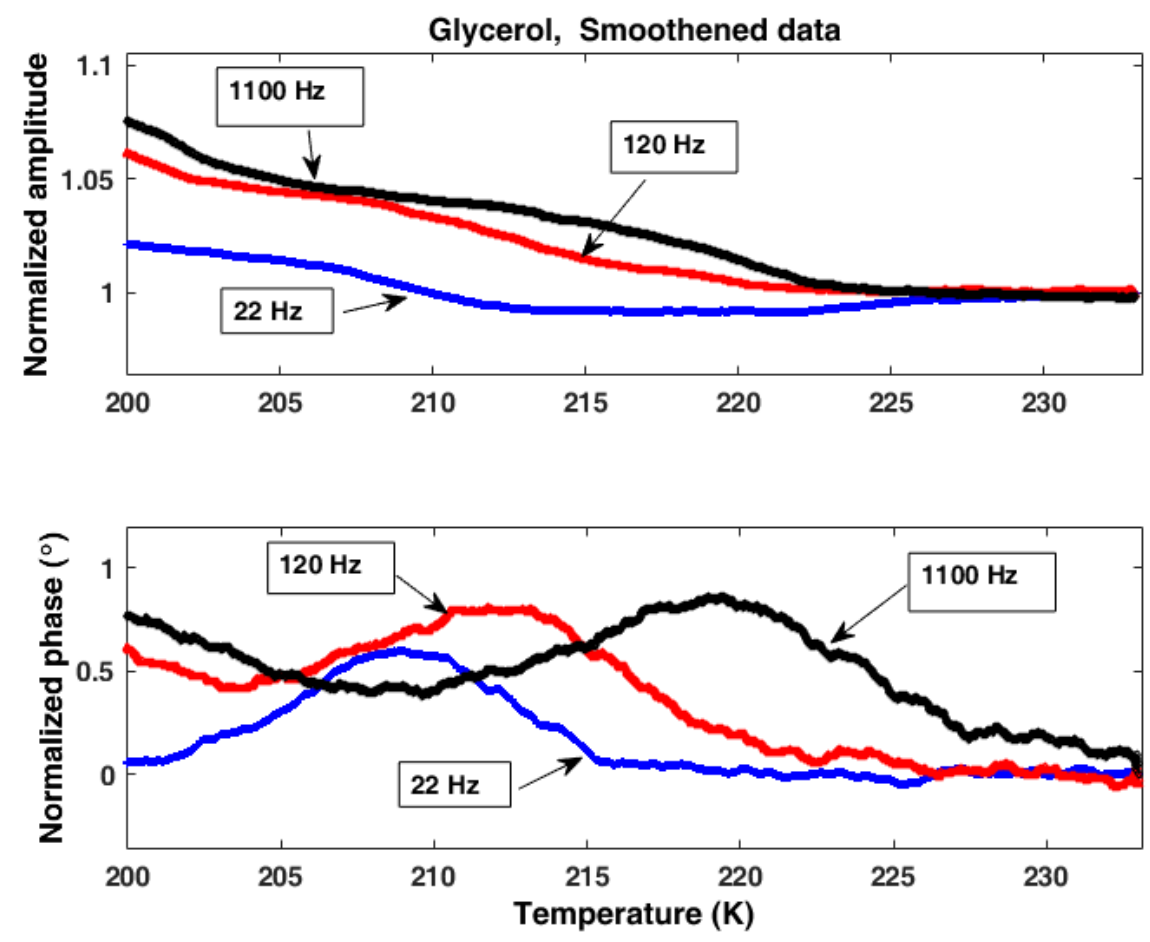

Figure 8: Normalized amplitude and phase of pure glycerol during a temperature scan at different frequencies using a $500 \mu m \mathrm{LiTaO}_{3}$ as sensor. 


\begin{tabular}{|c|c|c|c|c|c|c|c|c|}
\hline $\begin{array}{c}\mathrm{f} \\
(\mathrm{Hz})\end{array}$ & $\alpha$ & $\beta$ & $\begin{array}{c}e_{s_{\infty}}^{2} \\
\left(W^{2} s m^{-4} K^{-2}\right)\end{array}$ & $\begin{array}{c}\Delta e_{s}^{2}=e_{s 0^{-}}^{2} e_{s \infty}^{2} \\
\left(W^{2} s m^{-4} K^{-2}\right)\end{array}$ & $\begin{array}{c}\mathrm{B} \\
(\mathrm{K})\end{array}$ & $\begin{array}{c}T_{0} \\
(\mathrm{~K})\end{array}$ & $\begin{array}{c}\tau_{\infty} \\
(\mathrm{s})\end{array}$ & $\begin{array}{c}\tau_{\infty} \\
(\mathrm{s})\end{array}$ \\
\hline 22 & 0.98 & 0.52 & 212178 & 153545 & 1457 & 151 & $10^{-12.6735}$ & $10^{-12.67}$ \\
\hline 120 & 1.00 & 0.27 & 242098 & 295508 & 1855 & 151 & $10^{-15.3081}$ & $10^{-15.30}$ \\
\hline 1100 & 0.96 & 0.29 & 245387 & 326958 & 1205 & 169 & $10^{-13.5382}$ & $10^{-13.53}$ \\
\hline
\end{tabular}

Table 1: Best fitted HN and VFT parameters using 7-parameter fit from PPE experiments at different frequencies.

\begin{tabular}{|c|c|}
\hline$T_{g}(\mathrm{~K})$ mean $(\mathrm{PPE})$ & $T_{g}(\mathrm{~K})$ (literature) \\
\hline $195 \pm 6$ & $190^{a}, 191^{b}, 183.4^{c}$ \\
\hline
\end{tabular}

Table 2: $T_{g}$ of pure glycerol and their comparison with literature data, a[13], b[41], c[38].

\section{CONCLUSION}

A low temperature PPE instrumentation setup has been constructed and was used to study the glassy state determine the glass transition temperature of pure glycerol. PPE signal was analysed using Havriliak Negami function. VFT model was used for the temperature dependence of relaxation time $(\tau)$. A fit was performed on the temperature evolution of PPE signal to determine all the seven parameters. Once the VFT parameters were determined, the glass transition temperature $\left(T_{g}\right)$ of pure glycerol has been determined successfully by using the glass transition convention (relaxation time of 100s). Obtained results were This temperature was compared with literature and found to be in good agreement.

\section{Acknowledgment}

The authors would like to acknowledge CIEDIL industries, INNOCOLD and Aquimer for their support.

\section{References}

[1] A. A. Pronin, K. Trachenko, M. V. Kondrin, A. G. Lyapin, and V. V. Brazhkin. Nonlocal dielectric relaxation in glycerol. Phys. Rev. B - Condens. Matter Mater. Phys., 84:012201, 2011.

[2] D. Stefan and H. L. Williams. Molecular motions in bisphenol a polycarbonates as measured by pulsed NMR techniques. I. Homopolymers and copolymers. J. Appl. Polym. Sci., 18:1279, 1974.

[3] P. S. Gill, S. R. Sauerbrunn, and M. Reading. Modulated differential scanning calorimetry. J. Therm. Anal., 40:931, 1993.

[4] M. Reading, A. Luget, and R. Wilson. Modulated differential scanning calorimetry. Thermochim. Acta, 238:295, 1994. 
[5] S. Pittois, B. V. Roie, C. Glorieux, and J. Thoen. Static and dynamic thermal quantities near the consolute point of the binary liquid mixture aniline-cyclohexane studied with a photopyroelectric technique and adiabatic scanning calorimetry. J. Chem. Phys., 122: 024504, 2005.

[6] D. Dadarlat, V. Surducan, K. J. Riezebos, and D. Bicanic. A new photopyroelectric cell for thermal characterization of foodstuffs; Application to sugar systems. Instrum. Sci. Technol., 26:125, 1998.

[7] J. Mura, L. C.M. Miranda, M. L. Baesso, A. C. Bento, and A. F. Rubira. Piezo- and pyroelectric photothermal characterization of polymers as a function of temperature. $J$. Appl. Polym. Sci., 82:2607, 2001.

[8] E. H. Bentefour, C. Glorieux, M. Chirtoc, and J. Thoen. Broadband photopyroelectric thermal spectroscopy of a supercooled liquid near the glass transition. J. Appl. Phys., 93: 9610, 2003.

[9] G. S. Parks and W. A. Gilkey. Studies on glass. IV. Some viscosity data on liquid glucose and glucose-glycerol solutions. J. Phys. Chem., 33:1428, 1929.

[10] S. A. Brawer. Theory of relaxation in viscous liquids and glasses. J. Chem. Phys., 81: 954, 1984.

[11] L. Sjögren and W. Götze. Alpha relaxation near the glass transition. J. Non. Cryst. Solids, 131-133:153, 1991.

[12] F. Stickel, E. W. Fischer, and R. Richert. Dynamics of glass-forming liquids. I. Temperature-derivative analysis of dielectric relaxation data. J. Chem. Phys., 102:6251, 1995.

[13] R. Böhmer, K. L. Ngai, C. A. Angell, and D. J. Plazek. Nonexponential relaxations in strong and fragile glass formers. J. Chem. Phys., 99:4201, 1993.

[14] H. Bässler. Viscous flow in supercooled liquids analyzed in terms of transport theory for random media with energetic disorder. Phys. Rev. Lett., 58:767, 1987.

[15] I. Avramov. Viscosity in disordered media. J. Non. Cryst. Solids, 351:3163, 2005.

[16] J. C. Mauro, Y. Yue, A. J. Ellison, P. K. Gupta, and D. C. Allan. Viscosity of glass-forming liquids. Proc. Natl. Acad. Sci., 106:19780, 2009.

[17] J. C. Mauro. Through a glass, darkly: Dispelling three common misconceptions in glass science. Int. J. Appl. Glas. Sci., 2:245, 2011.

[18] A. Drozd-Rzoska, S. J. Rzoska, and M. Paluch. Universal critical-like scaling of dynamic properties in symmetry-selected glass formers. J. Chem. Phys., 129:184509, 2008.

[19] A. Drozd-Rzoska, S. J. Rzoska, S. Pawlus, J. C. Martinez-Garcia, and J. L. Tamarit. Evidence for critical-like behavior in ultraslowing glass-forming systems. Phys. Rev. E Stat. Nonlinear, Soft Matter Phys., 82:031501, 2010.

[20] G. A. López-Muñoz and J. A. Balderas-lópez. Photothermal characterization of citrus essential oils and their derivatives. Thermochim. Acta, 579:40-44, 2014. 
[21] A. O. Guimarães, F. A .L. Machado, E. C. da Silva, and A. M. Mansanares. Investigating thermal properties of biodiesel / diesel mixtures using photopyroelectric technique. Thermochim. Acta, 527:125-130, 2012.

[22] K. Strzalkowski, D. Dadarlat, M. Streza, and F. Firszt. On the optimization of experimental parameters in photopyroelectric investigation of thermal diffusivity of solids. Thermochim. Acta, 614:232-238, 2015.

[23] E. H. Bentefour, C. Glorieux, M. Chirtoc, and J. Thoen. Thermal relaxation of glycerol and propylene glycol studied by photothermal spectroscopy. J. Chem. Phys., 120:3726, 2004.

[24] R. Ivanov, E. Marin, P. D. Alaniz, J. J. Villa, and J. A. Bermejo Arenas. Self-normalized front photopyroelectric technique for thermal effusivity measurements in liquids. Thermochim. Acta, 599:8, 2015.

[25] K. E. Zachariassen. The mechanism of the cryoprotective effect of glycerol in beetles tolerant to freezing. J. Insect Physiol., 25:29, 1979.

[26] P. Boutron and A. Kaufmann. Stability of the amorphous state in the system waterglycerol-dimethylsulfoxide. Cryobiology, 15:93, 1978.

[27] M. Chirtoc, E. H. Bentefour, C. Glorieux, and J. Thoen. Development of the frontdetection photopyroelectric (FPPE) configuration for thermophysical study of glassforming liquids. Thermochim. Acta, 377:105, 2001.

[28] R. Ivanov, E. Marin, J. Villa, D. Alaniz, and E. Olvera. Self-normalized inverse photopyroelectric technique with viscous front window for thermal effusivity measurements in liquids. Thermochim. Acta, 655:124, 2017.

[29] D. Dadarlat, A. Frandas, M. Marinelli, F. Mercuri, and D. Bicanic. New photopyroelectric scheme suitable for phase-transition investigations: The front configuration with semitransparent sensor. Appl. Phys. A Mater. Sci. Process., 61:183, 1995.

[30] D. Dadarlat, H. Visser, and D. Bicanic. An improved inverse photopyroelectric cell for measurement of thermal effusivity : application to fatty acids and triglycerides. Meas. Sci. Technol., 6:1215, 1995.

[31] P. S. Jeon, J. H. Kim, H. J. Kim, and J. Yoo. The measurement of thermal diffusivities for semi-infinite solids using the photothermal displacement method. Thermochim. Acta, 494:65-70, 2009.

[32] A. H. Sahraoui, S. Longuemart, D. Dadarlat, S. Delenclos, C. Kolinsky, and J. M. Buisine. The application of the photopyroelectric method for measuring the thermal parameters of pyroelectric materials. Rev. Sci. Instrum., 73:2766, 2002.

[33] A. Oleaga, V. Shvalya, A. S. Sefat, and A. Salazar. Transport thermal properties of LiTaO3 pyroelectric sensor from $15 \mathrm{~K}$ to $400 \mathrm{~K}$ and its application to the study of critical behavior in EuCo2As2. Int. J. Thermophys., 37:4, 2016.

[34] N. O. Birge. Specific Heat Spectroscopy of glycerol and propylene glycol near glass transition. Phys. Rev. B, 34:1631, 1986. 
[35] S. Havriliak and S. Negami. A complex plane representation of dielectric and mechanical relaxation processes in some polymers. Polymer (Guildf)., 8:161, 1967.

[36] P. Dixon, L. Wu, S. R. Nagel, B. D. Williams, and J. Carini. Scaling of the relaxation of supercooled liquids. Phys. Rev. Lett., 65:1108, 1990.

[37] A. Puzenko, Y. Hayashi, Y. E. Ryabov, I. Balin, Y. Feldman, U. Kaatze, and R. Behrends. Relaxation dynamics in glycerol-water mixtures: I. Glycerol-rich mixtures. J. Phys. Chem. B, 109:6031, 2005.

[38] M. Cutroni, A. Mandanici, and L. De Francesco. Fragility, stretching parameters and decoupling effect on some supercooled liquids. J. Non. Cryst. Solids, 307-310:449, 2002.

[39] A. Salazar and A. Oleaga. On the piezoelectric contribution to the photopyroelectric signal. Rev. Sci. Instrum., 76:034901, 2005.

[40] Glycerine Producers' Association. Physical properties of glycerine and its solutions. New York, 1963.

[41] J. C. Martinez-Garcia, S. J. Rzoska, A. Drzozd-Rzoska, J. Martinez-Garcia, and J. C. Mauro. Divergent dynamics and the Kauzmann temperature in glass forming systems. Sci. Rep., 4:1, 2014. 